\title{
Characteristic of ZMYND10 Gene's Promoter Hypermethylation in Nasopharyngeal Carcinoma Biopsies from Vietnamese Patients
}

\author{
Thuan Duc Lao and Thuy Ai Huyen Le* \\ Department of Pharmaceutical and Medical Biotechnology, Faculty of Biotechnology, \\ Ho Chi Minh City Open University, Ho Chi Minh City, Vietnam; thuy.Iha@ou.edu.vn
}

\begin{abstract}
Silencing of tumor suppressor gene, which caused by the DNA hypermethylation, an epigenetic modification, has been reported to be involved in human various cancers, including nasopharyngeal carcinoma. The aims of this study were to identify and evaluate the hypermethylation frequency of the ZMYND10 promoter, which located at 3p21.3, in nasopharyngeal biopsies from Vietnamese patients by Nested Methylation Specific PCR (Nested MSP). In current study, ninety tumor biopsies and ninety healthy samples, which were obtained from Cho Ray hospital, were enrolled into study. As the results, the hypermethylation frequency of ZMYND10 gene promoter was more frequent in tumor biopsies. In detail, the hypermethylation frequency of ZMYND10 gene promoter were $81.11 \%$ (73 of 90 samples), and $45.56 \%$ ( 41 of 90 samples) for in NPC biopsies and non-cancerous specimens. A trend toward positive association was found between hypermethylation of ZMYND10 gene and nasopharyngeal carcinoma $(p<0.0007)$. Additionally, the high Odds ratio (OR) and Relative risk were observed $(\mathrm{OR}=5.13, \mathrm{RR}=2.49)(p<0.0001)$. In conclusion, our data suggested that the hypermethylation of ZMYND10 gene promoter is a significant in nasopharyngeal carcinoma in Vietnamese patients.
\end{abstract}

Keywords: Hypermethylation, Nasopharyngeal Carcinoma, Tumor Suppressor Gene, ZMYND10

\section{Introduction}

Nasopharyngeal Carcinoma (NPC) is a prevalent malignant tumor of nasopharynx has considered remarkably distinctive geographic and ethnic contribution, gravitating toward Southern Asia, especially in China and Vietnam ${ }^{1,2}$. According to statistics of Globocan (2012), the high prevalence of NPC cases was observed in reached to 4,931 cases $(\mathrm{ASR}=5.4 / 100,000)$ and deaths was 2,885 cases $(\mathrm{ASR}=3.3 / 100,000)$ in Vietnamese population ${ }^{3}$. For the past few years, many studies have been demonstrated that multiple risk factors, including Epstein-Barr virus infection, genetics/or epigenetic changes and environmental factor have been suggested to be strongly linked to
$\mathrm{NPC}^{4}$. Moreover, there is growing evidence demonstrating that the prevalent epigenetic changes, the hypermethylation of CpG islands in promoter regions of genes, the abnormalities at 3 p21.3 region, contribute to many cell processes in cell-cycle regulation, apoptosis, signal transduction, cell adhesion, etc., which leading to the inactivation or less expression of these TSGs involves in many human tumorigenesis including $\mathrm{NPC}^{5-7}$.

Involvement of the $3 \mathrm{p} 21.3$ disorder, such as the hypermethylation of tumor suppressor gene, in various cancers including Nasopharyngeal Carcinoma (NPC) has been reported previously ${ }^{8-11}$. Among several genes located at the 3 p21.3 region, such as RASSF1A, ZMYND10, PR2L, 101F6, PL6, etc., many studies have been suggested that

*Author for correspondence 
Zinc-finger, MYND-type containing 10 (ZMYND10), commonly called ZMYND10, spans $4.5 \mathrm{kbs}$ on $3 \mathrm{p} 21.3$, expression has been identified to be frequently downregulated in human NPC due to promoter hypermethylation. Previous studies shown that the frequencies of methylated ZMYND10 gene's promoter varied from $34.1 \%$ to $64.0 \%$ and $0 \%$ to $22.22 \%$ in primary NPC biopsy tissue and non-cancerous samples, including non-nasopharyngeal tissue, non-neoplastic nasopharyngeal epithelia, respectively ${ }^{12-14}$. Additionally, these alterations have been detected in NCP biopsies as well as the NPC cell lines ${ }^{10-13}$. The function of ZMYND10 protein has been considered as inhibitor of colony formation of cancer cells and found it could be activated by environmental stresses such as heat sock and its regulated by E2 $\mathrm{F}^{13,14}$. It is clear that the function of ZMYND10 gene is very important, and its reduced activity or loss of function by mechanisms such as hypermethylation is considered as a signal for cancer pathogenesis as well as an attractive target for developing therapeutic strategies towards NPC.

However, ZMYND10 promoter hypermethylation has not been investigated in NPC specimens from Vietnam. Therefore, the current study was the first case-control study, which aims to identify the profile of ZMYND10 promoter hypermethylation in a series of NPC samples which were collected from Vietnamese NPC patients and non-cancerous nasopharyngeal swab samples to reveal potential biomarkers for NPC.

\section{Materials and Methods}

\subsection{Ethics Statement}

Institutional Ethics Board approval was obtained from the Medical Ethics Committee of the Cho Ray Hospital, Ho Chi Minh City, Vietnam. (The decision number of the permission from Ethical committee: 516/BVCR-HDDD, Cho Ray Hospital, Ho Chi Minh City, Vietnam). All the samples used in this study were agreed by Cho Ray Hospital and obtained from all participants in this clinical trial. The patients are required to be agreed and sign on the consent forms.

\subsection{Samples Collection}

Ninety biopsy samples were collected from nasopharyngeal cancer patients, in Cho Ray Hospital, Ho Chi Minh City, Vietnam. All of those biopsies were collected from patients who were obeyed to ethical approval for study human samples, and patients agreed with purpose of the study. All the samples were submitted to histopathological diagnosis and confirmed NPC. In addition, ninety nasopharyngeal swab samples, which were collected from healthy donors used as as negative-nasopharyngeal carcinoma control.

\subsection{DNA Extraction, Bisulfite Modification}

Total of genomic DNA was isolated from biopsy or swab samples by phenol/chloroform method. Cells obtained from samples were lysed in lysis buffer $(10 \mathrm{mM}$ Tris- $\mathrm{HCl}$ $\mathrm{pH}=8,10 \mathrm{mM}$ EDTA, $150 \mathrm{mM} \mathrm{NaCl}, 2 \%$ SDS) containing Proteinase $\mathrm{K}(0.1 \mathrm{mg} / \mathrm{ml})$. Then, total of genomic DNA was isolated and purified by using standard phenol-chloroform and ethanol precipitation. The bisulfite conversion of $2 \mu \mathrm{g}$ genomic DNA was performed using EpiTect Bisulfite Kits (Qiagen). The final precipitation was eluted in a volume of $20 \mu \mathrm{l}$ and stored at $-20^{\circ} \mathrm{C}$ for further studies.

\subsection{Nested-Methylation Specific Polymerase Chain Reaction}

The methylation status of each promoter in samples were examined by two-steps nested PCR. In current study, firstly, the primers of stage 1 PCR were used for preceding amplification which recognize the bisulfite-modified template, notably, do not discriminate between methylated and unmethylated sequences. The sequence of the forward and reverse primers of stage 1 were 5'TTGGGAATTTAAATATTATG3', and 5'AACAACAATTCCAAATCTC3', respectively ${ }^{14}$. In stage 2 PCR, two pairs of primer were used to amplify the regions of interest. One pair recognized a sequence in which CpG sites were methylated (unmodified by bisulfite treatment). Other pair recognized a sequence in which CpG sites were unmethylated (modified to UpG treatment). The sequence of the methylated forward and reverse primers were 5' GCGGGTTAGAGATTCGTTC3', and 5'TCGAAACCGAAATCCGACG3', respectively. The sequence of the unmethylated forward and reverse primers were 5'GGTGGGTTAGAGATTTGTTT3', and 5'ATATCAAAACCAAAATCCAACA3', respectively ${ }^{14}$. Each stage of PCR was performed in a total of $15 \mu \mathrm{l}$ containing $3 \mu$ bisulfite-modified template DNA (in case of stage 1 PCR) or $3 \mu \mathrm{l}$ stage 1 PCR product (in case of stage 2 PCR), 0.75 unit iTaq DNA polymerase (Biorad), 0.5 $\mu \mathrm{M}$ each primer, $7.5 \mu \mathrm{lMyTaqTM}$ Mix (Bioline). Thermal 
cycling was initiated at $95^{\circ} \mathrm{C}$ for $5 \mathrm{~min}$, followed by 40 cycles of denaturation at $95^{\circ} \mathrm{C}$ for $30 \mathrm{sec}$, annealing at the $\mathrm{X}^{\circ} \mathrm{C}$ for $30 \mathrm{sec}$, extension at $72^{\circ} \mathrm{C}$ for $30 \mathrm{sec}$, and a final extension at $72^{\circ} \mathrm{C}$ for $10 \mathrm{~min}$ (Note: $\mathrm{X}^{\circ} \mathrm{C}$ was the specific annealing temperature for each specific methylated or unmethylated primer, $\mathrm{X}^{\circ} \mathrm{C}=50^{\circ} \mathrm{C}, 58^{\circ} \mathrm{C}$ and $64^{\circ} \mathrm{C}$ for Stage 1, methylated and unmethylated primers, respectively). Finally, the PCR products of the methylated and unmethylated were separated on $2 \%$ agarose gel and visualized by ethidium bromide staining. MSP products were sequencing to confirm the specificity of primers, examine the efficiency of bisulfite modification and the hypermethylation status of target gene.

\section{Statistical Analysis}

Statistical analyses were performed by Medcalc ${ }^{\circledR}$ Version 12.7.0.0. The average frequency of methylation was calculated. The association between hypermethylation of ZMYND10 and NPC were examined by using Chi-square test. A p-value $\leq 0.05$ was considered statistically significant. Moreover, the association between hypermethylation of ZMYND10 and risk of NPC was estimated by computing OR, RR and 95\% Confidence Intervals (CI).

\section{Results}

\subsection{Status of the Promoter Hypermethylation of ZMYND10}

The frequency of the promoter methylation of ZMYND10 in 90 nasopharyngeal biopsy samples and 90 non-cancer samples were examined by nested-MSP. Overall, the promoter frequencies for ZMYND10 in NPC samples and non-cancerous samples were $81.11 \%$ (73 of 90 samples), and $45.56 \%$ (41 of 90 samples), respectively. Conversely, the promoter unmethylation frequencies were $18.89 \%$ (17 of 90 samples), and $54.44 \%$ (49 of 90 samples) in NPC samples and healthy samples, respectively. Additionally, the $\mathrm{p}<0.0001$ indicated the methylation of ZMYND10 in NPC samples was found to be significant higher than in non-cancerous samples.

The MSP products of samples hypermethylation and/ or unmethylation in the promoter of ZMYND10 were observed all 180 samples, including 90 biopsies and 90 swabs samples (Figure 1). According to Figure 1, the MSP products of ZMYND10 in clinical samples were observed in the band of 231 bps and 235 bps length in case of methylation and unmethylation, respectively. The sequencing of samples hypermethylated promoter region of representative sample revealed a conversion of unmethylated Cytosine, but not methylated Cytosine (Figure 2). By sequencing, comparison between the non-bisulfite modified (Figure 2a) and bisulfite modified (Fig. 2b), all methylated Cytosines were unchanged, which were marked by square symbols. Otherwise, all the unmethylated Cytosines were totally changed into Thymine, which were indicated by triangle symbols in bisulfite sequence. Additionally, three methylated $\mathrm{CpG}$ sites were observed in methylated reverse primer, which were according to the primer designed.

\subsection{Odds Ratio, Relative Risk for Promoter Hypermethylation in ZMYND10 Gene}

In this study, the odds ratio and relative risk values were computed between NPC biopsy samples and non-can-

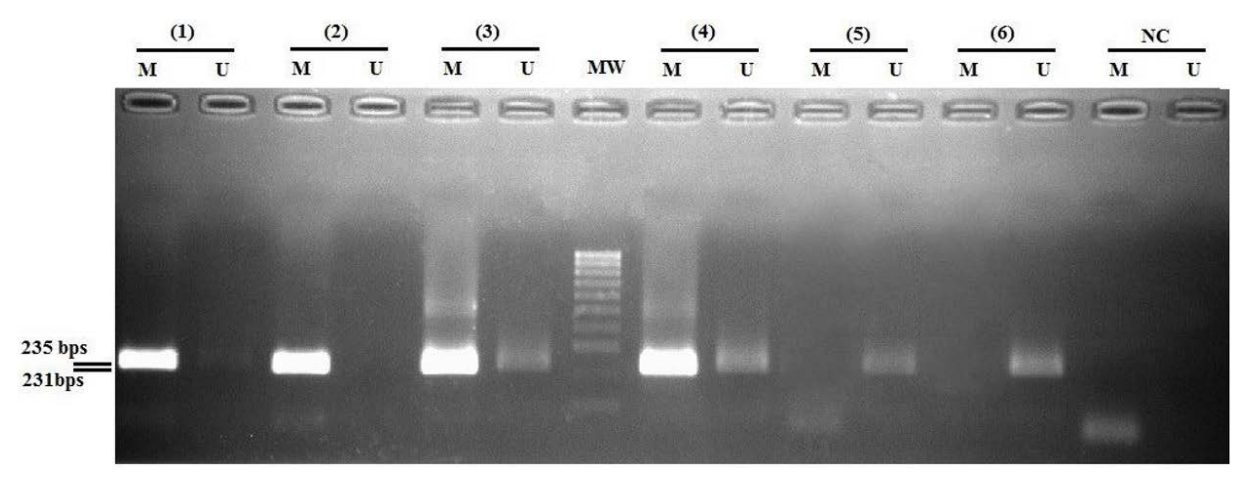

Figure 1. Methylated promoter of ZMYND10 gene was analyzed on some clinical samples by nested-MSP. (1) (2) (3) (4) NPC biopsy samples; non-cancerous sample; (5), (6) non-cancerous samples; NC: negative control; MW: molecular weight $100 \mathrm{bp}$ ladder. 


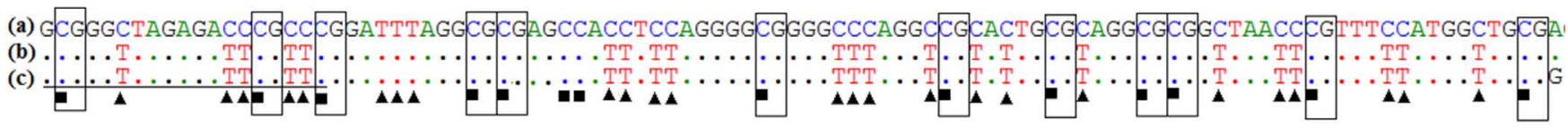

Figure 2. Sequencing profile of methylated ZMYND10 segment (Stage 2 PCR). CG sites were in flamed; Forward primer sequence is underlined; (a) DNA sequence was without bisulfite modified (Accession number: AC02481); (b) DNA sequence was bisulfite modified by Methprimer; (c) The ZMYND10 sequencing by using the forward primer.

cerous samples. The results show that, odds ratio, relative risk values were $5.13(95 \% \mathrm{CI}=2.62-10.04, p<0.0001)$ and $2.49(95 \% \mathrm{CI}=1.61-3.83, p<0.0001)$, respectively.

\section{Discussion}

Nasopharyngeal carcinoma is one of the commonly occurring cancers among Asian region, including Vietnam with the high prevalence ${ }^{3}$. Within the ambiguous symptoms such as hearing loss, nosebleeds, headache, trouble opening the mouth, etc., thus, most patients present with the stage III or IV cancer when diagnosed ${ }^{15,16}$, thus, it could be a challenge in early diagnosis. Therefore, to achieve favorable treatment and increasing of patient's survival, early diagnosis and prognosis are necessary to be appropriate managed. Previous studies have been shown that DNA hypermethylation plays an important role in human cancer development, including NPC. In particular, according to Lo and Huang (2012), alteration of the 3 p21.3 loci are highly prevalent in NPC with a combination of Loss of Heterozygosity (LOH) and aberrant promoter hypermethylation. ${ }^{17}$ In current study, we examined the methylation status of ZMYND10, which map to this 3 p21.3 region in NPC biopsy samples by Nested-MSP method. The combination both nested-PCR and MSP shows an advantage in the hypermethylation analysis by increased MSP sensitivity approximately 50-fold ${ }^{18}$. CpG islands are proven to be associated with tumor suppressor gene promoter and remain unmethylated in normal cell. Aberrant methylation of CpG islands is the common event in tumors cell subsequently loss of TSG function led to tumorigenesis. In our study, a high frequency of aberrant methylation in ZMYND10 promoter $(81.11 \%)$ in NPC biopsy sample was found, which showed higher frequency when compared to $66.0 \%-74.0 \%$ in the Chinese $\mathrm{NPC}^{12,13}$ and $34.1 \%$ in the Tunisian NPC samples ${ }^{14}$. Here, we also found that the hypermethylation of ZMYND10 promoter in 41 of 90 non-cancerous samples, counting for $45.56 \%$, was higher than previous studies, which com- pared to $0 \%$ to $22.22 \%$ in previous studies ${ }^{12-14}$. The high frequency of methylated ZMYND10 promoter in noncancerous samples could be explained that ZMYND10 may be methylated in age-related manner (in current study: the mean of age: 45 , varies from 15 to 82 ). The latter of which may conform to the hypothesis that the age-related methylation in normal samples which may lead to field defect in association with acquired predisposition to cancer ${ }^{12}$. However, we found the correlation between methylation of ZMYND10 gene promoter and NPC. The $\mathrm{p}$-value $(p<0.0001)$ pointed out the strongly significant statistical association between aberrant methylation of ZMYND10 gene and NPC. In fact, the function of ZMYND10 protein, its encoded protein, has been reported as inhibitor of colony formation of cancer cells, and found it could be activated by environmental stresses such as heat sock and its regulated by $\mathrm{E} 2 \mathrm{~F}^{13}$. Moreover, tumor suppressor ZMYND10 inhibits proliferation of NPC cells by regulation of cell cycle, c-Jun N-terminal kinase and the cyclin D1 promoter ${ }^{19}$. Recently, growing evidences proved that the loss of ZMYND10 expression was downregulated correlated with promoter hypermethylation $^{12,13,19}$. Therefore, it strongly suggested that ZMYND10 might be one of the important TSG candidates at this locus in NPC. The hypermethylation of ZMYND10 was significantly associated with an approximately 5.13-fold increase in NPC than compared to noncancerous samples $(\mathrm{OR}=5.13,95 \% \mathrm{CI}=2.62-10.04, \mathrm{p}<$ $0.0001)$ Concerning to $\mathrm{RR}$ value, it indicated that the risk of nasopharyngeal tumorigenesis significantly increased 2.49 times in the case within aberrant methylation of ZMYND10 gene promoter, leading to the inactivation of ZMYND10 $(\mathrm{RR}=2.49,95 \% \mathrm{CI}=1.61-3.83, \mathrm{p}<0.0001)$. Therefore, due to these results, we believed that aberrant methylation of ZMYND10 gene is a significant in nasopharyngeal carcinoma in Vietnamese patients, and the Nested-MSP method for ZMYND10 hypermethylation detection in NPC biopsy samples could to be considered as the promising biomarker that could be potentially used 
for diagnosis and prognostic purposes in Vietnam. It is noted that the discovery of hypermethylated TSGs has been detected not only in primary tumors, but also in serum, sputum, bodily fluids, saliva, etc. Therefore, as a next stage of our study, we intend to analyze the methylation status of $Z M Y N D 10$ gene in several non-invasive samples to develop the non-invasive biomarkers which will be easily applied in clinic, to prognosis and early diagnosis of NPC in Vietnamese population.

\section{Conclusion}

In summary, the results of this study showed a higher prevalence of ZMYND10 promoter hypermethylation in NPC biopsy samples, counting for $81.11 \%$. On the contrary, the low frequency of ZMYND10 promoter hypermethylation was found in healthy samples. Additionally, the significant correlation between candidate gene hypermethylation and human nasopharyngeal tumorigenesis, as well as the odds ratio and relative risk were found in the significant correlation, counting for 5.13 and 2.49, respectively were reported. And, the screening, which based on the detection of ZMYND10 promoter hypermethylation, will be an auspicious characteristic for early prognosis and diagnosis of NPC. In further study, the present findings require extension to numbers of many sources of sample in order to find out the potential non-invasive tumor markers for diagnosis and prognostic purposes in Vietnam.

\section{Acknowledgement}

We wish to express our thanks to the research project sponsored by Ho Chi Minh City Department of Science and Technology, Vietnam; Ho Chi Minh City Open University, HCMC, Vietnam. We also thank all the recruited participants in this work and all the staff members of Otorhinolaryngology in Cho Ray Hospital, Ho Chi Minh City, for collecting the samples used in these studies. We are thankful to Ngo Dong Kha, Thieu Hong Hue, Ho Ta Giap for their assistances.

\section{Author's Contribution}

Thuan LD 50\%; Thuy LHA 50\%. All authors read and approved the final manuscript.

\section{Ethics Approval and Consent to Participate}

All patients signed inform consent before entering into the study. No study drug or procedure was applied. This is an observational study.

\section{Conflict of interest}

The authors declared that they have no competing interests.

\section{References}

1. Sham JS, Wei WI, Zong YS, Choy D, Guo YQ, Luo Y, Lin $\mathrm{ZX}, \mathrm{Ng} \mathrm{MH}$, et al. Detection of subclinical nasopharyngeal carcinoma by fibreoptic endoscopy and multiple biopsy. Lancet. 1990; 335(8686):371-4. https:// doi.org/10.1016/0140-6736(90)90206-K

2. Chang ET, Adami HO. The enigmatic epidemiology of nasopharyngeal carcinoma. Cancer Epidemiol Biomarkers Prev. 2006; 15(10):1765-77. https://doi.org/10.1158/10559965.EPI-06-0353 PMid:17035381

3. GLOBOCAN. Estimated cancer incidence, mortality and prevalence worldwide in 2012. Available from: http://globocan.iarc.fr/Pages/fact_sheets_population.aspx

4. Hildesheim A, Levine PH. Etiology of nasopharyngeal carcinoma: A review. Epidemiol Rev. 1993; 15(2):466-85. https://doi.org/10.1093/oxfordjournals.epirev.a036130 PMid:8174667

5. Herman JG, Graff JR, Myöhänen S, Nelkin BD, Baylin SB. Methylation-specific PCR: A novel PCR assay for methylation status of CpG islands. Proc Natl Acad Sci U S A. 1996; 93(18):9821-6. https://doi.org/10.1073/pnas.93.18.9821 PMid:8790415 PMCid:PMC38513

6. Santini V, Kantarjian HM, Issa JP. Changes in DNA methylation in neoplasia: pathophysiology and therapeutic implications. Ann Intern Med. 2001; 134(7):573-86. https:// doi.org/10.7326/0003-4819-134-7-200104030-00011 PMid: 11281740

7. Challouf S, Ziadi S, Zaghdoudi R, Ksiaa F, Ben Gacem $\mathrm{R}$, Trimeche M. Patterns of aberrant DNA hypermethylation in nasopharyngeal carcinoma in Tunisian patients. Clin Chim Acta. 2012; 413(7-8):795-802. https://doi. org/10.1016/j.cca.2012.01.018 PMid:22296674

8. Kok K, Naylor SL, Buys CH. Deletions of the short arm of chromosome 3 in solid tumors and the search for suppressor genes. Adv Cancer Res. 1997; 71:27-92. https://doi. org/10.1016/S0065-230X(08)60096-2 
9. Ji L, Minna JD, Roth JA. 3p21.3 tumor suppressor cluster: prospects for translational applications. Future Oncol. 2005; 1(1):79-92. https://doi.org/10.1517/14796694.1.1.79 PMid:16555978

10. Cheng Y, Ho RL, Chan KC, Kan R, Tung E, Lung HL, et al. Anti-angiogenic pathway associations of the $3 \mathrm{p} 21.3$ mapped BLU gene in nasopharyngeal carcinoma. Oncogene. 2015; 34(32):4219-28. https://doi.org/10.1038/ onc.2014.353 PMid:25347745 PMCid:PMC4761643

11. Hesson LB, Cooper WN, Latif F. Evaluation of the 3p21.3 tumour-suppressor gene cluster. Oncogene. 2007; 26(52):7283-301. https://doi.org/10.1038/sj.onc.1210547 PMid:17533367

12. Liu XQ, Chen HK, Zhang XS, Pan ZG, Li A, Feng QS, et al. Alterations of BLU, a candidate tumor suppressor gene on chromosome 3p21.3, in human nasopharyngeal carcinoma. Int J Cancer. 2003; 106(1):60-5. https://doi.org/10.1002/ ijc.11166 PMid:12794757

13. Qiu GH, Tan LK, Loh KS, Lim CY, Srivastava G, Tsai ST, et al. The candidate tumor suppressor gene BLU, located at the commonly deleted region 3p21.3, is an E2F-regulated, stress-responsive gene and inactivated by both epigenetic and genetic mechanisms in nasopharyngeal carcinoma. Oncogene. 2004; 23(27):4793-806. https://doi.org/10.1038/ sj.onc.1207632 PMid:15122337

14. Ayadi W, Karray-Hakim H, Khabir A, Feki L, Charfi S, Boudawara T, et al. Aberrant methylation of p16, DLEC1,
BLU and E-cadherin gene promoters in nasopharyngeal carcinoma biopsies from Tunisian patients. Anticancer Res. 2008; 28(4B):2161-7. PMid:18751390

15. Epstein JB, Jones CK. Presenting signs and symptoms of nasopharyngeal carcinoma. Oral Surg Oral Med Oral Pathol. 1993; 75(1):32-6. https://doi. org/10.1016/0030-4220(93)90402-P

16. Hao SP, Tsang NM, Chang KP, Ueng SH. Molecular diagnosis of nasopharyngeal carcinoma: detecting LMP-1 and EBNA by nasopharyngeal swab. Otolaryngol Head Neck Surg. 2004; 131(5):651-4. https://doi.org/10.1016/j. otohns.2004.04.013 PMid:15523443

17. Lo KW, Huang DP. Genetic and epigenetic changes in nasopharyngeal carcinoma. Semin Cancer Biol. 2002; 12(6):451-62. https://doi.org/10.1016/ S1044579X02000883

18. Palmisano WA, Divine KK, Saccomanno G, Gilliland FD, Baylin SB, Herman JG, et al. Predicting lung cancer by detecting aberrant promoter methylation in sputum. Cancer Res. 2000; 60(21):5954-8. PMid:11085511

19. Zhang X, Liu H, Li B, uang P, Shao J, He Z. Tumor suppressor BLU inhibits proliferation of nasopharyngeal carcinoma cells by regulation of cell cycle, c-Jun N-terminal kinase and the cyclin D1 promoter. BMC Cancer. 2012; 12:267. https://doi.org/10.1186/1471-2407-12-267 PMid:22727408 PMCid:PMC3585814 